\title{
Differential Tick Burdens May Explain Differential Borrelia afzelii and Borrelia garinii Infection Rates among Four, Wild, Rodent Species in Hokkaido, Japan
}

\author{
Kyle R. TAYLOR ${ }^{1)}$, Ai TAKANO'), Satoru KONNAI ${ }^{3)}$, Michito SHIMOZURU ${ }^{1)}$, Hiroki KAWABATA ${ }^{2)}$ and \\ Toshio TSUBOTA ${ }^{1) *}$ \\ ${ }^{1)}$ Laboratory of Wildlife Biology and Medicine, Graduate School of Veterinary Medicine, Hokkaido University, Kita 18 Nishi 9, Sapporo, \\ Hokkaido 060-0818, Japan \\ 2) Department of Bacteriology-I, National Institute of Infectious Diseases, 1-23-1 Toyama, Shinjuku-ku, Tokyo 162-8640, Japan \\ ${ }^{3)}$ Laboratory of Infectious Diseases, Graduate School of Veterinary Medicine, Hokkaido University, Kita 18 Nishi 9, Sapporo, Hokkaido \\ 060-0818, Japan
}

(Received 1 October 2012/Accepted 25 January 2013/Published online in J-STAGE 8 February 2013)

ABSTRACT. The ecologies of Lyme disease Borrelia spp. are very specific to location, as they are dependent upon the spirochete species and genotypes, the vectors and the host vertebrates present. In Hokkaido, Japan, where two human pathogenic, Lyme disease Borrelia spp. are present, and human cases are reported annually, the ecologies have been poorly studied. Our goal was to determine whether variation in borrelial infection rates among rodent species sharing an environment, is due to immunological or ecological differences. To this end, we examined the relationships between tick burden and borrelial infection, by including examination of agreement between nested PCR, as a test for infection, and serology, as a test for exposure. We collected 868 rodents, comprised of four species commonly found in Hokkaido, and tested for infection rates with Borrelia spp. using PCR for the borrelial flaB gene, seroprevalence of Borrelia afzelii and Borrelia garinii using ELISA, and attachment of ticks by direct counts. We noted a correlation between differential nymph and larval burdens and the borrelial infection rates found among the four rodent species. Furthermore, there was significant correlation between infection and seroprevalence of $B$. afzelii and B. garinii $(P<0.01)$, between infection and Ixodes persulcatus nymph burden $(P<0.01)$, and between seroprevalence and $I$. persulcatus nymph burden $(P<0.01)$. The close agreement among rodent species seroprevalences with infection rates and tick burdens suggest the differences in infection rates of Borrelia spp. may largely be a direct consequence of differential exposure to vectors.

KEY WORDS: Borrelia, Ixodes, vector-borne, wild rodents, zoonosis.

doi: 10.1292/jvms.12-0439; J. Vet. Med. Sci. 75(6): 785-790, 2013

Lyme disease is an important and emerging, zoonotic disease throughout much of the northern hemisphere. Human cases are on the rise in many endemic regions of the world. However, in Japan, cases have remained relatively stable over the past decade [13]. In Japan, human Lyme disease cases are caused mainly by Borrelia garinii, but also Borrelia afzelii, with the most common culprits being $B$. garinii strains carried by rodents [20]. All known, Lyme Borrelia spp. pathogenic to humans in Japan are transmitted by the vector tick, Ixodes persulcatus [22].

Rodents are likely the most important reservoir species of human pathogenic Borrelia spp., in Hokkaido, an area with particularly high Lyme disease risk in Japan [13], and $I$. persulcatus ticks are the vectors. Therefore, a clear and full understanding of the relationships between rodent species, and the tick is a pre-requisite in being able to predict changes in risk to humans with changes in the environment-either human induced or otherwise. However, studies focusing on these relationships have been few in Japan, and assumptions have been largely made based on research performed

*Correspondence to: Tsubota, T., Laboratory of Wildlife Biology and Medicine, Graduate School of Veterinary Medicine, Hokkaido University, Kita 18 Nishi 9, Sapporo, Hokkaido 060-0818, Japan. e-mail: tsubota@vetmed.hokudai.ac.jp

(C)2013 The Japanese Society of Veterinary Science in the United States and Europe, on different Borrelia spp. and genotypes, different vectors and different host species. Since Lyme disease cases have not been increasing in Japan, however, the situation is likely not the same as in other locations. Therefore, we endeavored, with this study, to describe the relationships of the spirochetes, vectors and host species specific to Hokkaido, Japan. The main question we address is whether differential infection rates among rodent species sharing the same niche are due to immunological differences or ecological differences.

\section{MATERIALS AND METHODS}

Rodent sampling was performed in two locations in Hokkaido, Japan, as described in a previous study [21]. Briefly, Sherman traps were used to catch rodents in central Hokkaido, near Shimizu and in eastern Hokkaido, near Shari for three to six days in each location from May through September, 2010 and 2011. Sampling locations were chosen based on convenience and rodent abundance. Captured rodents were anesthetized with isoflurane (Dainippon Sumitomo Pharma Co., Ltd., Osaka, Japan) and were euthanized by cervical dislocation prior to organ sampling. The urinary bladder of each rodent was collected, since it has been reported to be a sensitive organ for determining Lyme disease Borrelia spp. infections [9]. Feeding ticks attached to the neck and head were collected and counted. Serum was col- 
lected by spinning down fresh blood samples placed in 1.5 $\mathrm{m} l$ centrifuge tubes at approximately $1,000 \mathrm{rpm}$ for $10 \mathrm{~min}$. All samples were stored at -20 or $-80^{\circ} \mathrm{C}$ until extraction of DNA. All rodent sampling was performed using methods approved by the Animal Care and Use Committee of Hokkaido University (Approval No. JU1105).

Rodents were identified to species based on morphologic characteristics [1, 14] and were classified as adults or subadults based on previously described criteria [21].

Urinary bladder DNA was extracted, and nested polymerase chain reaction (PCR) and direct sequencing were performed to check for the presence of the borrelial flagellin B $(f l a B)$ gene as previously described [21]. Sequencing results were compared to reference sequences recorded in GenBank for identification to species (B. garinii strain 20047-greater than $98 \%$ similarity and B. afzelii strain VS461, B. japonica strain HO14 and B. miyamotoi strain HT31-each greater than 99\% similarity. Accession numbers: D86617, D63365, D82852 and D43777, respectively).

For serological examination of borrelial infections, ELISA was performed on serum samples using a recomWell Borrelia IgG kit (Mikrogen GmbH, Neuried, Germany), which contains the recombinant antigens p100, OspC, VlsE and p18, and which is capable of identifying exposure to Borrelia burgdorferi sensu stricto, B. garinii and B. afzelii. Although the kit was originally designed for use with canine serum, we replaced the anti-dog IgG secondary antibody contained in the kit with peroxidase-labeled goat anti-mouse $\operatorname{IgG}(\mathrm{H}+\mathrm{L})$ (Kirkegaard \& Perry Laboratories, Inc., Gaithersburg, MD, U.S.A.), which has been used for rodents of the Apodemus and Myodes genera [8, 19], diluted to a final concentration of $0.01 \mu \mathrm{g} / \mathrm{ml}$. The negative cut-off was defined as 3 standard deviations from the mean absorbance readings of negative controls, as is convention for similar studies [8, 19]. Negative controls were serum taken from six C57/BL6 mice (Japan SLC, Inc., Hamamatsu, Japan) with Borrelia spp. negative $I$. persulcatus ticks attached. All samples with absorbance values higher than the negative cut-off were considered ELISApositive. Four positive controls, C57/BL6 mice infected with $B$. garinii, were also run, and all had absorbance values well above the negative cut-off.

Using the recomLine Borrelia IgG kit (Mikrogen $\mathrm{GmbH}$ ), which includes the recombinant borrelial antigens, p100, VlsE, p58, p41, p39, OspA, OspCs and p18s, we performed immunoblotting to evaluate the appropriateness of our antimouse IgG immunoglobulin as a secondary antibody for the ELISA. Using three ELISA-positive and three ELISAnegative sera for each of the four species tested in this study: Apodemus argenteus, Apodemus speciosus, Myodes rufocanus and Myodes rutilus. We performed the immunoblotting according to the manufacturer's protocol, except that we substituted our anti-mouse IgG mentioned above, diluted to a final concentration of $0.001 \mu \mathrm{g} / \mathrm{ml}$, as the secondary antibody.

All statistical analyses were performed using GraphPad InStat and Prism (GraphPad Software, Inc., La Jolla, CA, U.S.A.). Tests of correlation between two parameters were performed using Fisher's exact test, while correlation analy- ses among the four rodent species were all performed using the Kruskal-Wallis test with Dunn's multiple comparisons test.

\section{RESULTS}

A total of 868 rodents were trapped and included in this study. Four species were represented: A. argenteus $(\mathrm{n}=135)$, A. speciosus $(\mathrm{n}=448)$, M. rufocanus $(\mathrm{n}=196)$ and $M$. rutilus $(\mathrm{n}=89)$. The urinary bladder was collected and tested for the presence of the borrelial $f a B$ gene from all rodent samples. ELISA was performed on 391 of the collected rodent samples (A. argenteus, $\mathrm{n}=53$, A speciosus, $\mathrm{n}=226$, . rufocanus, $\mathrm{n}=78$ and $M$. rutilus, $\mathrm{n}=34$ ), the number of attached ticks was counted on 804 of the collected rodent samples ( $A$. argenteus, $\mathrm{n}=124$, A. speciosus, $\mathrm{n}=419$, . rufocanus, $\mathrm{n}=176$ and $M$. rutilus, $\mathrm{n}=85)$, and both ELISA and feeding tick counts were available for 364 rodent samples (A. argenteus, $\mathrm{n}=48$, A. speciosus, $\mathrm{n}=216, M$. rufocanus, $\mathrm{n}=69$ and $M$. rutilus, $\mathrm{n}=31)$.

The results for PCR of the $f l a B$ gene are listed in Table 1. In order to compare PCR results with results for the ELISA, which does not differentiate between $B$. afzelii and $B$. garinii positive samples, we combined the PCR positive samples from these two Borrelia spp. into a single PCR positive group, omitting $B$. japonica and $B$. miyamoto $i$ positive samples, for all subsequent testing and analyses. Under this re-classification, there were 127 PCR positive samples and 741 PCR negative samples $($ A. argenteus, pos. $=5$, neg. $=130$; A. speciosus, pos. $=101$, neg. $=347 ;$ M. rufocanus, pos. $=10$, neg. $=186 ;$ M. rutilus, pos. $=11$, neg. $=78)$. There was a statistically significant difference in prevalence among species $(P<0.01)$, with $A$. argenteus and $M$. rufocanus having lower infection rates than $A$. speciosus ( $P<0.01$ for each). The prevalence of $B$. afzelii and/or $B$. garinii infection by species is shown in Table 1 and Fig. 1A. There was no significant difference in infection rates of rodents collected from the two areas.

For seroprevalence comparisons by ELISA, 140 of the 391 serum samples we tested had antibody responses greater than the cut-off value we set $(A$. argenteus, pos. $=4, A$. speciosus, pos. $=103$, M. rufocanus, pos. $=21$ and $M$. rutilus, pos.=12). There was a statistically significant difference among species, with $A$. argenteus $(P<0.01)$ and $M$. rufocanus $(P<0.05)$ showing lower seroprevalences than $A$. speciosus. The seroprevalences of each species are shown in Table 2 and Fig. 1B.

Twelve ELISA-positive sera (three from each of four rodent species) used in this study showed reactivity to borrelial antigens by immunoblotting, whereas all 12 ELISA-negative sera (also three from each of four rodent species) tested were also negative by immunoblotting (data not shown). This suggests that the ELISA used in this study detects rodent antibody against borrelial antigens for all four species evaluated in this study.

A total of 2,083 ticks were collected from 804 rodents (A. argenteus, 148 ticks from 124 samples, A. speciosus, 1,380 ticks from 419 samples, M. rufocanus, 375 ticks from 
Table 1. DNA detection of Borrelia spp. by flaB-PCR in rodents

\begin{tabular}{|c|c|c|c|c|c|c|}
\hline Rodent species & No. tested & B. afzelii & B. garinii & B. japonica & B. miyamotoi & Total \\
\hline A. argenteus & 135 & $0(0 \%)$ & $5(3.7 \%)$ & $0(0 \%)$ & $1(0.7 \%)$ & $6(4.4 \%)$ \\
\hline A. speciosus & 448 & $41(9.1 \%)$ & $69(15.4 \%)$ & $0(0 \%)$ & $10(2.2 \%)$ & $111(24.8 \%)^{\mathrm{a})}$ \\
\hline M. rufocanus & 196 & $4(2.0 \%)$ & $7(3.6 \%)$ & $10(5.1 \%)$ & $2(1.0 \%)$ & $22(11.2 \%)^{\mathrm{a})}$ \\
\hline M. rutilus & 89 & $5(5.6 \%)$ & $8(9.0 \%)$ & $2(2.2 \%)$ & $0(0 \%)$ & $\left.12(13.5 \%)^{\mathrm{a}, \mathrm{b}}\right)$ \\
\hline \multirow[t]{3}{*}{ Total } & 868 & $50(5.8 \%)$ & $89(10.2 \%)$ & $12(1.4 \%)$ & $13(1.5 \%)$ & $151(17.4 \%)^{\mathrm{a}, \mathrm{b})}$ \\
\hline & Total tested & \multicolumn{2}{|c|}{ B. afzelii and/or B. garinii positive } & & & \\
\hline & 868 & \multicolumn{2}{|c|}{$127(14.6 \%)$} & & & \\
\hline
\end{tabular}

a) B. afzelii and B. garinii mixed samples present ( $A$. speciosus $=9$, ,. rufocanus $=1, M$. rutilus $=2$, Total=12). b) One $B$. garinii and B. japonica mixed sample present.
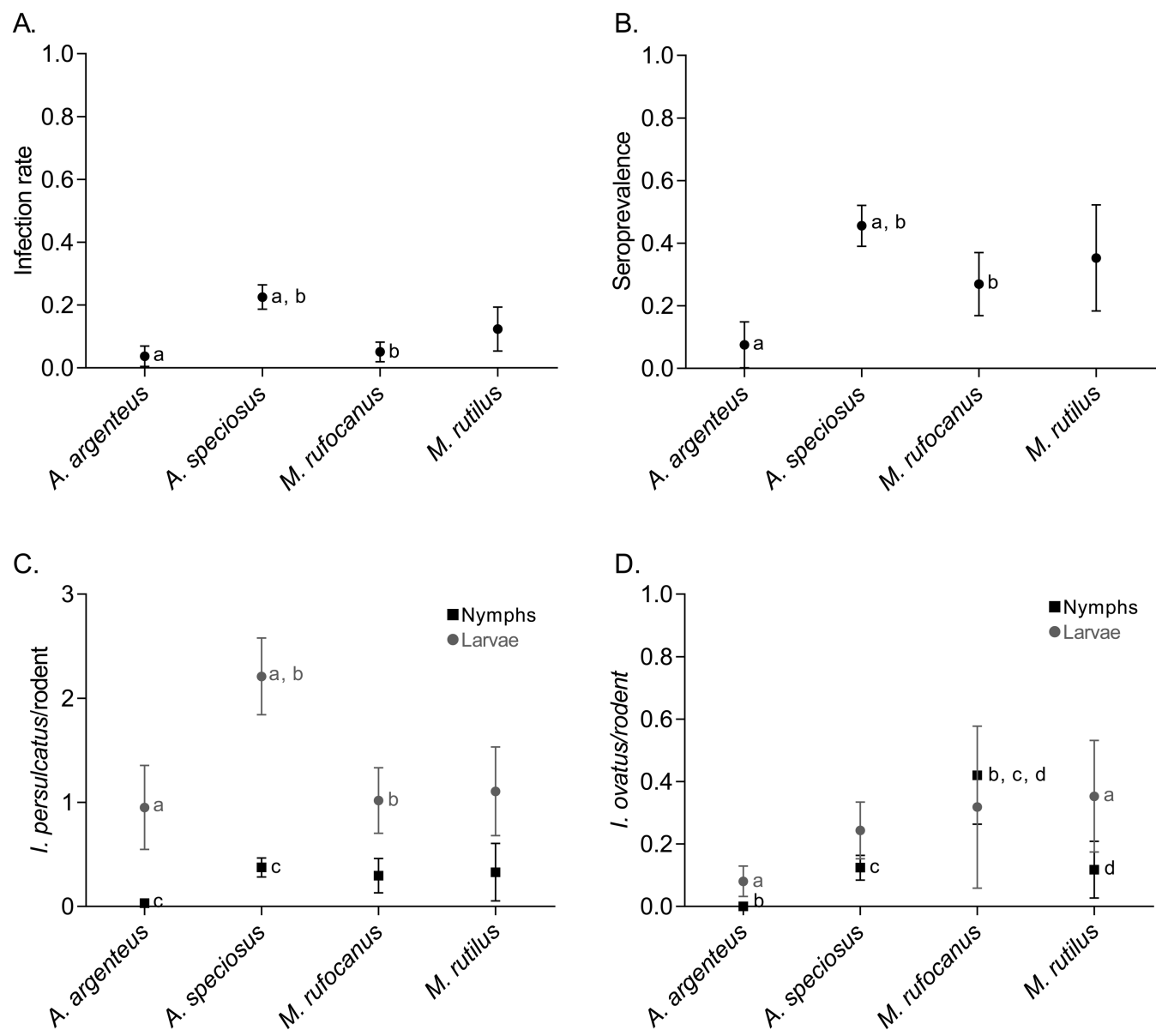

Fig. 1. A. Combined infection rates of B. afzelii and B. garinii by rodent species. Significant differences between rodent species are represented by a and b. B. Rates of exposure to B. afzelii and/or B. garinii by rodent species (ELISA-positive over total for each rodent species). Significant differences between rodent species are represented by a and b. C. Average number of I. persulcatus nymphs and larvae attached per rodent for each species (total number of ticks by stage over total rodents of each species). Significant differences for nymphs are represented by $\mathrm{c}$, and for larvae, a and b. D. Average number of I. ovatus nymphs and larvae. Significant differences for nymphs are represented by b, c and d, and for larvae, a only. All error bars represent $95 \%$ confidence intervals.

176 samples and M. rutilus, 180 ticks from 85 samples). The largest portion of the total belonged to I. persulcatus larvae at $1,317(63.2 \%)$, and $241(11.6 \%)$ of the total ticks recovered were I. persulcatus nymphs. Ixodes ovatus larvae and nymphs accounted for $198(9.5 \%)$ and $136(6.5 \%)$, respectively. Only one unidentified adult Ixodes sp. tick was collected.

A total of just four I. persulcatus nymphs were collected 
Table 2. Seroprevalence to Lyme disease borreliae in rodents by ELISA

\begin{tabular}{lcc}
\hline Rodent species & No. tested & ELISA-positive \\
\hline A. argenteus & 53 & $4(7.5 \%)$ \\
A. speciosus & 226 & $103(45.6 \%)$ \\
M. rufocanus & 78 & $21(26.9 \%)$ \\
M. rutilus & 34 & $12(35.3 \%)$ \\
\hline Total & 391 & $140(35.8 \%)$ \\
\hline
\end{tabular}

a) B. afzelii and B. garinii mixed samples present (A. speciosus $=9$, M. rufocanus $=1$, M. rutilus $=2$, Total=12). b) One B. garinii and $B$. japonica mixed sample present.

from $124 \mathrm{~A}$. argenteus samples (range: 0 to 2 per rodent), 157 nymphs were collected from 419 A. speciosus (range: 0 to 7 per rodent), 52 nymphs were collected from 176 M. rufocanus (range: 0 to 8 per rodent) and 28 nymphs were collected from $85 \mathrm{M}$. rutilus (range: 0 to 8 per rodent). There was a statistically significant difference among rodent species, with $A$. argenteus $(P<0.01)$ having fewer I. persulcatus nymphs attached than $A$. speciosus.

One hundred and eighteen I. persulcatus larvae were collected from $124 \mathrm{~A}$. argenteus samples (range: 0 to 14), 926 larvae were collected from 419 A. speciosus samples (range: 0 to 22), 179 larvae were collected from $176 \mathrm{M}$. rufocanus samples (range: 0 to 11) and 94 larvae were collected from 85 M. rutilus samples (range: 0 to 14 ). There was a statistically significant difference among rodent species, with $A$. argenteus and $M$. rufocanus burdened by fewer I. persulcatus larvae than $A$. speciosus $(P<0.01$ for both). The average numbers of $I$. persulcatus nymphs and larvae attached to each species are shown in Fig. 1C.

Fifty-two I. ovatus nymphs were collected from 419 A. speciosus (range: 0 to 11 per rodent), 74 nymphs were collected from $176 \mathrm{M}$. rufocanus samples (range: 0 to 21 per rodent) and 10 nymphs were collected from $85 \mathrm{M}$. rutilus (range: 0 to 5 per rodent). No I. ovatus nymphs were collected from any of the $124 \mathrm{~A}$. argenteus samples. There was a statistically significant difference among species, with $M$. rufocanus burdened by significantly more nymphs than all other species $(P<0.01$ for each) (Fig. 1D). Ten I. ovatus larvae were collected from $124 \mathrm{~A}$. argenteus samples (range: 0 to 1), 102 larvae were collected from 419 A. speciosus samples (range: 0 to 22), 55 larvae were collected from 176 M. rufocanus samples (range: 0 to 11) and 29 larvae were collected from $85 \mathrm{M}$. rutilus samples (range: 0 to 14). There was a statistically significant difference among species $(P<0.05)$, with $M$. rutilus carrying a larger burden of I. ovatus larvae than $A$. argenteus $(P<0.01)$. The average numbers of $I$. ovatus nymphs and larvae attached to each species are shown in Fig. 1D.

There was a significant difference in seroprevalences of adults and sub-adults, with proportionally more adults seropositive, for three species: $A$. speciosus, $P<0.01$, relative risk $=2.350$ (confidence interval $[\mathrm{CI}]: 1.890$ to 2.921 ) M. rufocanus, $P<0.05$, relative risk $=2.630$ (CI: 1.300 to 5.323 ) and $M$. rutilus, $P<0.05$, relative risk $=3.800$ (CI: 1.242 to 11.622). There was no significant difference between adults
Table 3. Contingency tables for borrelial PCR, seroprevalence against Borrelia spp. by ELISA, and Ixodes persulcatus nymph attachment

A. ELISA and PCR

\begin{tabular}{lccc}
\hline \multirow{2}{*}{ PCR (B. afzelii-B. garinii) $)$} & \multicolumn{3}{c}{ ELISA } \\
\cline { 2 - 4 } & Positive & Negative & Total \\
\hline Positive & 48 & 7 & 55 \\
Negative & 92 & 244 & 336 \\
\hline Total & 140 & 251 & 391 \\
\hline PCR positi
\end{tabular}

PCR positive samples are significantly more likely to be seropositive than seronegative (Fisher's Exact test, $P<0.01$ ).

B. Nymph attachment and ELISA

\begin{tabular}{lccc} 
& \multirow{2}{*}{ Nymphs } & \multicolumn{3}{c}{ ELISA } \\
\cline { 2 - 4 } & Positive & Negative & Total \\
\hline With & 27 & 16 & 43 \\
Without & 104 & 217 & 321 \\
\hline Total & 131 & 233 & 364 \\
\hline
\end{tabular}

Rodents with nymphs attached are significantly more likely to be seropositive than seronegative (Fisher's Exact test, $P<0.01$ ).

C. Nymph attachment and PCR

\begin{tabular}{lccc}
\hline \multirow{2}{*}{ Nymphs } & \multicolumn{3}{c}{ PCR (B. afzelii-B. garinii) } \\
\cline { 2 - 4 } & Positive & Negative & Total \\
\hline With & 38 & 76 & 114 \\
Without & 68 & 622 & 690 \\
\hline Total & 106 & 698 & 804 \\
\hline
\end{tabular}

Rodents with nymphs attached are significantly more likely to be PCR positive for either B. afzelii or B. garinii (Fisher's Exact test, $P<0.01$ ).

and sub-adults for $A$. argenteus.

The PCR results for $B$. miyamotoi and $B$. japonica were each compared to seroprevalence, yet neither showed statistically significant correlation.

Correlation between samples PCR positive for $B$. afzelii and/or $B$. garinii and seropositive samples was statistically significant for all rodent species combined $(P<0.01)$, with samples being more likely to test positive for PCR if ELISA was positive. The contingency table is shown in Table 3A.

The number of ticks attached to rodents was also compared to seroprevalence. There were statistically significant relationships between seropositive samples and both $I$. persulcatus nymph and larval attachment, with rodents more likely to be seropositive if they were parasitized by either $I$. persulcatus nymphs or larvae (seroprevalence to I. persulcatus nymph attachment, $P<0.01$, relative risk $=1.938$ [CI: 1.466 to 2.562]; seroprevalence to $I$. persulcatus larval attachment, $P<0.05$, relative risk=1.443 [CI: 1.096 to 1.900 ]) (Table 3B). Furthermore, there was a statistically significant correlation between PCR and attachment of either I. persulcatus nymphs or larvae, with rodents being more likely to be infected with B. afzelii and/or B. garinii if they were parasitized by either I. persulcatus nymphs or larvae (PCR to I. persulcatus nymph attachment, $P<0.01$, relative risk $=3.382$ [CI: 2.398 to 4.771 ], and PCR to I. persulcatus larval attachment, $P<0.01$, relative risk $=2.229$ [CI: 1.537 to 3.234$]$ ) $($ Table $3 \mathrm{C})$. 
Finally, the correlation between the attachment of I. persulcatus nymphs and larvae was examined. Nymphs and larvae were more likely to be found on the same rodent samples than not $(P<0.01$, relative risk $=1.789$ [CI: 1.553 to 2.082]).

\section{DISCUSSION}

It is well documented that different host species inhabiting the same environment can have different prevalences of Borrelia spp. infections among their populations [5, 10, 11]. Our PCR results re-affirm that this holds true in Hokkaido, Japan, and our data corroborate a previous study performed in Hokkaido [12]. It has even been shown that different rodent species can have different infection intensities for the same Borrelia spp. [15]. However, the reasons for these differences are unclear. Moreover, the large body of research on Borrelia spp. suggests that it is difficult to make generalizations across locales with different Borrelia spp. and genotypes, vectors and hosts. Therefore, we attempted to clarify what factors affect Borrelia spp. infection rates across species in Hokkaido, Japan, by comparing B. afzelii and $B$. garinii infection rates, determined by PCR, to seroprevalence, determined by ELISA, and tick attachment, determined by direct counts and species identification.

The correlation between seroprevalence and PCR for $B$. afzelii and B. garinii was statistically significant for three of four rodent species and in total. Although significant correlation was not shown for $A$. argenteus, we believe this is a consequence of the extremely small number of PCR positive samples, and we suspect we would see significant correlation with a larger sample size. No significant correlation was noted between seroprevalence and PCR for either B. japon$i c a$ or $B$. miyamotoi, suggesting that the ELISA could detect antibodies to B. afzelii and B. garinii, as expected, but not to $B$. japonica or B. miyamotoi. Moreover, the fact that adult rodents were more likely to be seropositive than sub-adults agrees with our previous study, which showed that a larger proportion of adults were infected with $B$. afzelii and B. garinii than were sub-adults [21]. Furthermore, immunoblotting indicated that the anti-mouse IgG we used as a secondary antibody was satisfactorily capable of reacting with borrelial antibodies of all four species in our study. In light of these results, we believe the ELISA provides sufficient means for evaluating exposure to $B$. afzelii and/or B. garinii.

The aggregation we saw in this study, of $I$. persulcatus larvae and nymphs on the same hosts, is in agreement with previous studies on Ixodes ricinus and Ixodes scapularis [2, 16]. Given this knowledge, we make the assumption that trends noted for larvae are also attributable to nymphs, and, therefore, a significant difference in attachment of nymphs between $A$. speciosus and $M$. rufocanus would likely be seen with a larger sample size. This is important, given the fact that $B$. afzelii and $B$. garinii are not vertically transmitted within the vector, and therefore, rodents do not acquire the spirochetes from larvae [17]. Furthermore, biased aggregation of feeding ticks on individual rodents [2] may help explain the good correlations between I. persulcatus nymph attachment and seroprevalence and infection, given that the latter two can theoretically be positive even after ticks have fallen off.

The correlations noted for tick burden and seroprevalence and infection with $B$. afzelii and/or B. garinii are strongly suggestive of a direct relationship between the three parameters. Some studies have suggested differential infection rates between rodent species may be due to host immune response differences against the spirochetes $[6,7]$. For example, one study has suggested that seroprevalence of borrelial exposure between two rodent species was higher in the species with the higher tick burden, while infection rate was actually higher in the species with the lesser burden - a discrepancy in exposure and infection that is, perhaps, best explained by immune response differences [8]. However, since, in our study, PCR results and seroprevalence are highly correlated, although differential immune responses directed toward B. afzelii and/or B. garinii still may affect infection rates, we suggest that the level of exposure to ticks, which would not be affected by such immunity, is likely a larger factor in Hokkaido.

Concerning differential immunity against ticks, one study suggested that some species develop resistance to the vector with repeated exposure, thereby reducing attachment time, engorgement and the tick burden of I. ricinus larvae [3]. Since our study does not directly evaluate this possible interaction, the differential tick burdens, and therefore, the differential exposure and infection rates could still be a result of such immune responses. However, we propose the possibility that with such a mechanism, rodents might still have ample chance to seroconvert and/or become persistently infected prior to the development of appropriate immunity against ticks. This, however, would, theoretically, reduce correlation between tick burden and seroprevalence and infection, which would not agree with our current results.

It is possible that either host behavior or tick preference or both leads to the differences in tick attachment. One research group suggested that the incongruent densities of questing nymphs and larvae and the numbers of those attached to hosts were consistent with a non-passive encounter model, meaning that the behavior of either the ticks or the hosts causes strong bias in tick attachment [18]. For example, among the four rodent species examined in this study, $A$. argenteus, which has the smallest tick burden, is the only partially arboreal species [14]. It is even possible that this species is partially arboreal in order to evade ticks, as one study has indicated that some species may avoid areas with large densities of questing ticks [4]. Different grooming habits of rodents do not fully explain the differences in tick attachment because, although compared to A. speciosus, $M$. rufocanus had fewer I. persulcatus ticks attached, the species had significantly more $I$. ovatus nymphs attached than all other species and M. rutilus had more I. ovatus larvae attached than any other species, although the difference was only significant compared to $A$. argenteus. This discrepancy is suggestive of different niches or hunting behavior of tick species, which may result in host preference.

Given that all rodents included in this study were collected on the same trapping grids, the species clearly share overlap- 
ping niches, yet there are clear differences in infection rates with and exposure to $B$. afzelii and B. garinii among different rodent species. Furthermore, our data suggest that those differences are largely a direct result of differences in tick burden. The causes of differences in tick burdens, however, remain unclear, and require further examination. Still, our study has provided new insight into the interactions of the spirochetes, vectors and hosts specific to Hokkaido, Japan.

ACKNOWLEDGMENTS. The authors thank Mr. Hidenori Nishizawa for his help in sampling, Drs. Satoshi Ohdachi and Jiro Arikawa for use of their traps, Dr. Hisashi Abe for instruction on rodent identification and aging, and Ms. Kozue Sato and Ms. Chieko Sugimori of the National Institute of Infectious Diseases for their help in cloning and related DNA work. This research was funded in part by the Mitsui \& Co., Ltd. Environment Fund, in part by a grant for research on emerging and reemerging infectious diseases from the Japan Ministry of Health, Labor and Welfare, and by the Global COE Program "Establishment of International Collaboration Centers for Zoonosis Control,” MEXT, Japan.

\section{REFERENCES}

1. Abe, H. 1994. A Pictoral Guide to the Mammals of Japan, Tokai University Press, Tokyo.

2. Brunner, J. L. and Ostfeld, R. S. 2008. Multiple causes of variable tick burdens on small-mammal hosts. Ecology 89: 2259-2272. [Medline] [CrossRef]

3. Dizij, A. and Kurtenbach, K. 1995. Clethrionomys glareolus, but not Apodemus flavicollis, acquires resistance to Ixodes ricinus $\mathrm{L}$, the main European vector of Borrelia burgdorferi. Parasite Immunol. 17: 177-183. [Medline] [CrossRef]

4. Fritzsche, A. and Allan, B. F. 2012. The ecology of fear: host foraging behavior varies with the spatio-temporal abundance of a dominant ectoparasite. EcoHealth 9: 70-74. [Medline] [CrossRef]

5. Humair, P. F., Rais, O. and Gern, L. 1999. Transmission of Borrelia afzelii from Apodemus mice and Clethrionomys voles to Ixodes ricinus ticks: differential transmission pattern and overwintering maintenance. Parasitology 118: 33-42. [Medline] [CrossRef]

6. Kurtenbach, K., Dizij, A., Seitz, H. M., Margos, G., Moter, S. E., Kramer, M. D., Wallich, R., Schaible, U. E. and Simon, M. M. 1994. Differential immune responses to Borrelia burgdorferi in European wild rodent species influence spirochete transmission to Ixodes ricinus L. (Acari: Ixodidae). Infect. Immun. 62: 5344-5352. [Medline]

7. Kurtenbach, K., Sowell, H., Ogden, N. H., Randolph, S. E. and Nuttall, P. A. 1998. Serum complement sensitivity as a key factor in Lyme disease ecology. Infect. Immun. 66: 1248-1251. [Medline]

8. Kybicova, K., Kurzova, Z. and Hulinska, D. 2008. Molecular and serological evidence of Borrelia burgdorferi sensu lato in wild rodents in the Czech Republic. Vector Borne Zoonotic Dis. 8: 645-652. [Medline] [CrossRef]

9. Masuzawa, T., Beppu, Y., Kawabata, H., Yanagihara, Y., Iwa- moto, Y., Shimizu, T. and Johnson, R. C. 1992. Experimental Borrelia burgdorferi infection of outbred mice. J. Clin. Microbiol. 30: 3016-3018. [Medline]

10. Marsot, M., Sigaud, M., Chapuis, J. L., Ferquel, E., Cornet, M. and Vourc'h, G. 2011. Introduced Siberian chipmunks (Tamias sibiricus barberi) harbor more-diverse Borrelia burgdorferi sensu lato genospecies than native bank voles (Myodes glareolus). Appl. Environ. Microbiol. 77: 5716-5721. [Medline] [CrossRef]

11. Mather, T. N., Wilson, M. L., Moore, S. I., Ribeiro, J. M. and Spielman, A. 1989. Comparing the relative potential of rodents as reservoirs of the Lyme disease spirochete (Borrelia burgdorferi). Am. J. Epidemiol. 130: 143-150. [Medline]

12. Nakao, M. and Miyamoto, K. 1993. Reservoir competence of the wood mouse, Apodemus speciosus ainu, for the Lyme disease spirochete, Borrelia burgdorferi, in Hokkaido, Japan. Jap. J. Sanit. Zool. 44: 69-84.

13. National Institute of Infectious Diseases. 2011. Lyme disease 2006 to 2010. IASR 32: 216-228. [cited 2012 September 11] Available from http://idsc.nih.go.jp/iasr/32/378/inx378-j.html (in Japanese).

14. Ohdachi, S. D., Ishibashi, Y., Iwasa, M. A. and Saitoh, T. 2009. The Wild Mammals of Japan, Mammalogical Society of Japan, Kyoto.

15. Raberg, L. 2012. Infection intensity and infectivity of the tickborne pathogen Borrelia afzelii. J. Evol. Biol. 25: 1448-1453. [Medline] [CrossRef]

16. Randolph, S. E., Miklisov, D., Lysy, J., Rogers, D. J. and Labuda, M. 1999. Incidence from coincidence: patterns of tick infestations on rodents facilitate transmission of tick-borne encephalitis virus. Parasitology 118: 177-186. [Medline] [CrossRef]

17. Richter, D., Debski, A., Hubalek, Z. and Muatuschka, F. R. 2012. Absence of Lyme disease spirochetes in larval Ixodes ricinus ticks. Vector Borne Zoonotic Dis. 12: 21-27. [Medline] [CrossRef]

18. Schmidt, K. A., Ostfeld, R. S. and Schauber, E. M. 1999. Infestation of Peromyscus leucopus and Tamias striatus by Ixodes scapularis (Acari: Ixodidae) in relation to the abundance of hosts and parasites. J. Med. Entomol. 36: 749-757. [Medline]

19. Stefancikova, A., Bhide, M., Pet'ko, B., Stanko, M., Mosansky, L., Fricova, J., Derdakova, M. and Travnicek, M. 2004. AntiBorrelia antibodies in rodents: important hosts in ecology of Lyme disease. Ann. Agric. Environ. Med. 11: 209-213. [Medline]

20. Takano, A., Nakao, M., Masuzawa, T., Takada, N., Yano, Y., Ishiguro, F., Fujita, H., Ito, T., Ma, X., Oikawa, Y., Kawamori, F., Kumagai, K., Mikami, T., Hanaoka, N., Ando, S., Honda, N., Taylor, K., Tsubota, T., Konnai, S., Watanabe, H., Ohnishi, M. and Kawabata, H. 2011. Multilocus sequence typing implicates rodents as the main reservoir host of human-pathogenic Borrelia garinii in Japan. J. Clin. Microbiol. 49: 2035-2039. [Medline] [CrossRef]

21. Taylor, K. R., Takano, A., Konnai, S., Shimozuru, M., Kawabata, H. and Tsubota, T. 2013. Borrelia miyamotoi infections among wild rodents show age and month independence and correlation with Ixodes persulcatus larval attachment in Hokkaido, Japan. Vector Borne Zoonotic Dis. 13: 92-97. [Medline] [CrossRef]

22. Yanagihara, Y. and Masuzawa, T. 1997. Lyme disease (Lyme borreliosis). FEMS Immunol. Med. Microbiol. 18: 249-261. [Medline] [CrossRef] 